\title{
esthétique et sitologie : amélioration du paysage urbain et rural
}

\author{
Rapport général de \\ A. Milliès-Lacroix
}

Monsieur le Président, Mesdames, Messieurs,

Introduire des notions de sitologie et des critères de jugement fondés sur l'esthétique dans des disciplines telles que la géologie ou la mécanique des sols et des roches, ce n'est, à première vue, pas banal... Et pourtant, les spécialistes des sols et des roches sont des acteurs à part entière dans l'acte de construire et dans le développement de la cité. Comme tels, la qualité architecturale de l'urbanisme et de l'environnement les concerne aussi.

C'est aujourd'hui une banalité de rappeler que cette qualité architecturale passe d'abord par les fondations et par une meilleure adaptation des structures à la nature du sol: c'est la partie cachée de l'iceberg, la plus importante peut-être, celle par où peut périr l'édifice.

C'est par ailleurs le ministre de l'Équipement et du Cadre de Vie, à l'occasion de la cérémonie d'ouverture du $26^{\circ}$ Congrès Géologique International à Paris, qui soulignait la place de choix qu'occupent les sciences de la terre dans l'aménagement harmonieux de notre milieu naturel : comment elles favorisent l'utilisation rationnelle d'un bien de plus en plus rare, l'espace, et quel rôle elles jouent dans la planification de l'aménagement du territoire.

Ces sciences nous permettent d'évaluer les conséquences de nos projets et de réduire ou de supprimer celles qui seraient néfastes.

Elles contribuent à enrichir notre vie sociale en facilitant l'organisation de nos centres urbains.

Elles participent à la réhabilitation et à la reconstitution des paysages dénaturés par certaines activités industrielles, notamment extractives.

Elles aident enfin à préserver nos biens et à assurer la sécurité des personnes et permettent de nous guider en imposant aux ouvrages des règles de localisation, les mettant ainsi à l'abri de ces risques.
Ce sont ces sciences qui autorisent éventuellement une modification de notre environnement, dans le respect des équilibres fondamentaux, c'est-à-dire de l'écologie. Elles permettent ainsi d'éviter un immobilisme ou une interdiction systématique de remodeler un paysage, paysage qui n'a d'ailleurs bien souvent de naturel que le nom.

Ceci est important: tout aménagement n'est pas forcément une atteinte néfaste au milieu.

L'histoire en forme de parabole rappelée par lan Mc Harg dans son ouvrage $~$ Design with Nature $n$ n'est pas à prendre au pied de la lettre. Pour la petite histoire, on se souvient qu'il s'agit d'un des premiers cosmonautes, dans l'espace, et qui s'émerveille du spectacle offert à sa vue, avec la couleur émeraude des algues et de la végétation à la surface de la Terre et des Océans. Se rapprochant de la planète, il prend conscience des taches noirâtres ou brunâtres qui commencent à enserrer la verdure, à la façon des tentacules d'une pieuvre. En blêmissant, le cosmonaute réalise qu'il s'agit des cités et des aménagements de l'homme et s'écrie alors: "L'être humain est-il une maladie planétaire, à l'image d'une moisissure ou d'un cancer? „

Ce sont les sciences de la terre, je l'espère, qui permettront de dégager une évolution plus esthétique et plus harmonieuse, et d'abord par un recours systématique à la sitologie.

II n'est pas facile de définir un terme qui, pour beaucoup encore, reste un néologisme. On sait du moins qu'il ne s'agit pas de biologie cellulaire. Pour ce qui nous concerne et sans sophistication excessive, on doit pouvoir trouver un consensus, en définissant la sitologie comme l'étude des sites et des paysages, en vue d'une meilleure adaptation des ouvrages aux contraintes physiques, sur l'emprise de ces ouvrages et sur leur périphérie, c'est-à-dire par rapport à l'environnement. En d'autres termes, cela pourrait être «l'intégration au paysage . 
La sanction, dans ce domaine, est le jugement des utilisateurs, des contemporains, de la postérité.

Les critères d'évaluation, tenant compte notamment de l'esthétique, sont malaisés à apprécier, car soumis aux caprices de la mode et de l'évolution sociale.

II n'est pas évident de savoir quels sont les bâtiments ou les ouvrages que le temps consacrera, permettant leur intégration exemplaire dans le patrimoine immobilier de la Nation. Une destruction éventuelle de ces ouvrages, devenus «monuments historiques" par exemple, sera ressentie aujourd'hui comme une atteinte grave à "l'environnement .

Une approche différente, adaptée à notre société de consommation, vise à l'obsolescence calculée des constructions, pour ne pas risquer une atteinte durable au milieu: nul doute que les entreprises de travaux soient acquises à cette conception de l'art de bâtir.

II reste entendu que la notion de site n'est pas restrictive et qu'à la limite, avec ses connotations de paysage, elle participe de l'environnement et se confond avec lui.

Très schématiquement, on peut illustrer cette notion de la manière suivante (fig. 1) :

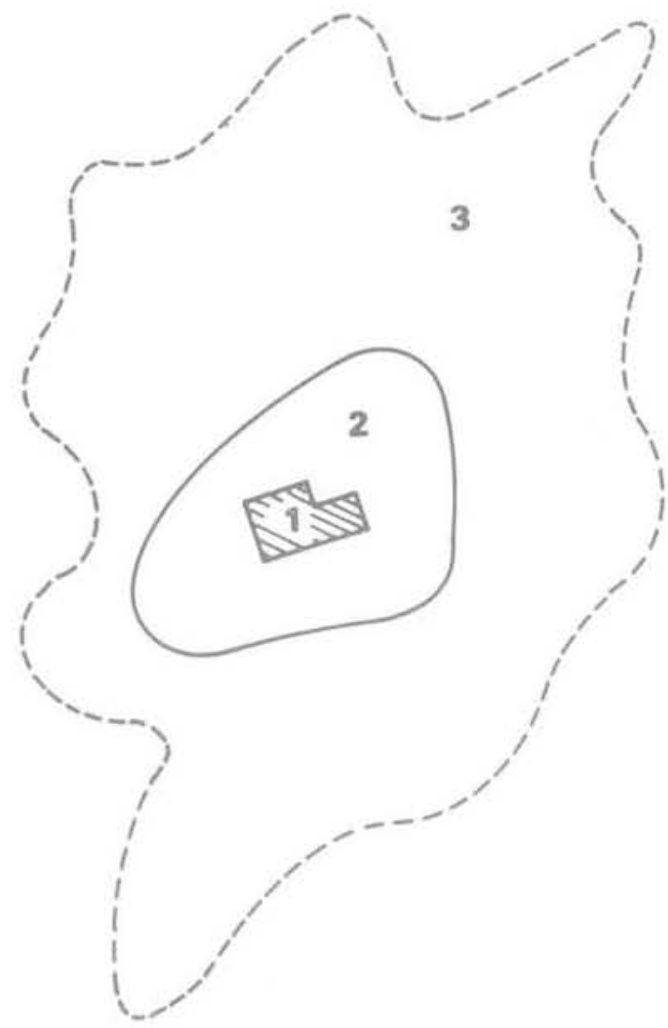

Fig. 1 Site d'un projet et contraintes physiques d'environnement

1 -Zone des contraintes "in situ», sur l'emprise de l'ouvrage (superficie occupée par un bâtiment par exemple)

2 -Zone où s'exercent des interactions directes, avec l'impact du projet sur la périphérie et les contraintes supplémentaires induites sur le projet par son environnement

3-Zone où les impacts sont plus difficiles à évaluer, avec des incidences plus floues, susceptibles de varier dans le temps et dans l'espace (risque de pollution accidentelle, par exemple)
- De fait, il y a bien le site stricto sensu, avec le terrain d'emprise bien déterminé d'un ouvrage ou d'une opération d'aménagement.

- En limite du projet, l'environnement immédiat, avec les u riverains" de l'opération.

- Au-delà, l'environnement général, susceptible d'être très étendu lorsqu'on met en jeu les impacts visuels ou les risques de pollution par les eaux ou l'atmosphère.

A ces trois échelles de prise en compte du site et de l'environnement peuvent correspondre des approches très différentes au niveau des études et de la conception des ouvrages.

Dans le premier cas, c'est l'adaptation classique du projet aux difficultés locales du site, pour tenir compte par exemple de l'existence de cavités dans le sous-sol ou de couches d'argiles compressibles à l'emplacement d'un bâtiment d'habitation.

Dans le second cas, l'adaptation du projet à l'environnement immédiat montre souvent une interaction : la réalisation d'une fouille profonde, en milieu urbain dense, aura un impact sur l'environnement mais devra aussi prendre en compte en retour les contraintes nées par exemple de l'existence de bâtiments vétustes en périphérie ou de la nécessité de maintenir la circulation, d'où mise en œuvre de paroi moulée, tirants d'ancrage, butons, écran d'étanchéité par injections..

Dans le troisième cas, touchant à un horizon plus étendu, les interactions seront généralement moins évidentes, avec des impacts également plus difficiles à évaluer, et plus particulièrement pour ce qui concerne les impacts visuels; l'adaptation se traduira par exemple par le choix d'une solution d'ouvrage semi-enterré, s'inscrivant plus harmonieusement dans le paysage, ceci pour profiter parallèlement du curage d'une couche tourbeuse superficielle.

Une autre catégorie de projets, évoquée dans quelques communications, est représentée par les ouvrages exécutés spécifiquement pour pallier les effets d'une pollution, et non rattachés à un projet d'aménagement particulier.

Pour en revenir au premier cas, on concoit que les géotechniciens se soient, de tous temps, préoccupés des contraintes physiques ayant un impact direct et évident sur les structures projetées, pour des raisons de coût et de risques : il s'agit là, en quelque sorte, des " servitudes" du site par rapport à un projet, implanté par exemple sur un versant instable.

Ce n'est pas un hasard si les marécages, les zones inondables, les talus instables, les terrains souscavés... ont été soit délaissés, soit colonisés tardivement, sous la pression de l'expansion démographique et urbaine.

La prise en compte en termes d'impact du projet sur l'environnement, avec les modifications éventuelles apportées aux études, à la conception et à la réalisation de ce projet, est une attitude de généralisation plus récente, renforcée en France par la loi du 10 juillet 1976 sur la protection des espaces naturels et par son décret d'application du 12 octobre 1977. II est bon de rappeler, à ce propos, que l'analyse réglementaire de "l'état initial du site et de son environnement ", prévue à l'article 2 de la loi du 10 juillet, est d'abord un problème à aborder dans l'optique des Sciences de la Terre. 
II y a là sans conteste un créneau important pour la géologie appliquée, surtout si l'on considère que les contraintes physiques généralement prises en compte dans les études d'impact concernent: la géologie stricto sensu, le climat, I'hydrologie et I'hydrogéologie, la topographie, la pédologie, la flore, la faune et le paysage.

A vrai dire, parmi les techniciens du sol, nombreux sont les précurseurs, qui, dans le passé, ont participé à la construction de la cité, collaborant avec les architectes et les ingénieurs pour une amélioration du cadre bâti.

Le "constructeur" pouvait alors réunir en une seule personne les compétences du géologue, de l'architecte et de l'ingénieur, et la démarche naturaliste des premiers temps s'est très souvent traduite par une recherche particulière dans la qualité du site et l'intégration au paysage.

C'est donc aujourd'hui, en quelque sorte, un retour aux sources, plus marqué peut-être chez le géologue, qui a toujours maintenu le contact avec le terrain et, partant, avec la Nature.

Au reste, avant même la création du Comité Français de Géologie de I'Ingénieur, nombreux étaient les géologues pour estimer que la première démarche, pour un aménageur, devait être l'étude comparative de plusieurs sites, comprenant, à côté des aspects sociologiques, économiques, climatologiques et de vocation des sols, une reconnaissance géologique et géotechnique autorisant un choix rationnel (1).

Dans cette reconnaissance, la prévision des modifications apportées au milieu naturel par les aménagements projetés (tassements du fait de pompages, inondations par suite de l'urbanisation et de la modification concomitante du coefficient de ruissellement...) est un élément d'appréciation indispensable.

C'est un point de vue voisin qui est exprimé par le groupe de travail constitué à l'initiative de J. Kérisel, rapporteur, à l'occasion du Symposium National * Sol et sous-sol et sécurité des constructions", tenu à Cannes en octobre 1973 (thème III: «Étude et définition des domaines d'intervention obligatoire d'un spécialiste des sols avant toute autorisation de travaux d'aménagement et en particulier avant délivrance du permis de construire n): L'analyse générale des contraintes physiques des opérations d'aménagement démontre la nécessité d'une "étude du site, c'est-àdire d'une étude géotechnique dépassant en général l'emprise de l'ouvrage».

La démarche va jusqu'à suggérer une " servitude pour l'étude du site ", comparable au " tour d'échelle ", pour faciliter les accès et rendre possible cette étude géotechnique.

Allant plus loin encore, ce groupe de travail propose une procédure d'intervention obligatoire du géotechnicien "en fonction de trois types de critères, liés respectivement au site, aux travaux d'exécution et aux caractéristiques de l'ouvragen. Dans ce contexte, l'étude géotechnique serait imposée pour tous les ouvrages publics et semi-publics.

(1) Arnould M. (1969) " Aspects géologiques des problèmes d'urbanisme - Bull. liaison Labo. Routiers Ponts et Chaussées $n^{\circ} 41$, pp. $93-100$.
Dans son allocution d'ouverture à ce même Symposium National de Cannes, C. Guillemin demandait déjà aux participants à l'acte de construire d'avoir toujours " présent à l'esprit que les réalisations futures doivent s'intégrer dans leur cadre naturel en préservant au maximum la physionomie de la planète, en évitant de détériorer de façon irrémédiable [...] ces paysages, sans respect d'un patrimoine qui est notre propriété à tous $"$.

II n'est pas inutile de rappeler ici qu'une des premières manifestations internationales de tout premier plan, concernant la géologie de l'environnement, a été organisée à la suggestion du regretté Dr. R. Wolters, secrétaire général de L'A. I. G. I. (Association Internationale de Géologie de l'Ingénieur): la section 13, représentant la Géologie de l'Ingénieur au $25^{\circ}$ Congrès géologique international organisé à Sydney, recevait des communications de douze pays sur le thème intitulé "contribution de la géologie à la gestion de l'environnement".

D'autres manifestations marquantes telles que les Journées d'Études d'Orléans (février 1978) consacrées aux * Recherches réalisées dans le cadre de l'environnement *, ou le Colloque national de Lyon (mars 1979) sur le thème de la connaissance du sous-sol et de l'aménagement urbain, ont facilité une prise de conscience par la profession, qui se sent de plus en plus concernée.

Au demeurant, cette orientation des Sciences de la Terre n'est pas isolée. On constate aujourd'hui :

- Un effort très important consenti en France pour l'environnement, avec une part sérieusement accrue dans les budgets de l'État, des collectivités et des entreprises.

- Une émergence accélérée des «produits " d'environnement, malgré la conjoncture récessionniste.

- Une adaptation des entreprises et des bureaux d'études à ce développement des produits d'environnement et d'abord au niveau des stratégies de groupe avec, par exemple, la définition de nouvelles entités fonctionnelles spécifiquement chargées de l'environnement.

Concrètement, l'engagement du géotechnicien pour une qualité accrue de l'urbanisme et pour une amélioration du paysage urbain et rural se situe à plusieurs niveaux:

Au niveau des études préalables, en vue d'un diagnostic ou d'une assurance de faisabilité, avec une campagne de reconnaissance préliminaire débordant généralement l'emprise proprement dite des ouvrages.

Au niveau de la conception des ouvrages, en concertation avec l'architecte ou le maître d' cuvre, pour permettre à ces derniers de mieux appréhender les conséquences des différents choix techniques. dans la solution de base et les solutions variantes.

Au niveau de l'exécution du projet, en accompagnement des différentes phases du chantier (terrassements, fouilles, rabattement de nappe, tirants d'ancrage, mise en place de butons...); ce suivi d'exécution allant parfois jusqu'à l'arbitrage, en cas de litiges entre les entreprises et le maître d'ouvrage.

Au niveau de la maintenance de l'ouvrage ou du suivi de l'étude d'environnement, avec par exemple le contrôle du tassement des fondations. 


\section{Présentation des communications}

Les communications ayant été publiées avant les journées géotechniques, il ne sera pas utile d'aller très loin dans l'analyse.

Parmi les cinq communications suscitées par le premier sous-thème, toutes ne répondent pas forcément, dans leur intégralité, aux préoccupations que je viens d'exposer.

Globalement, ces travaux montrent bien cependant cette prise en compte croissante des spécialistes des sols et des roches pour une meilleure intégration des ouvrages dans leur site naturel.

En fonction des sujets abordés, je pense que nous pouvons distinguer quatre démarches dans les communications présentées :

- Un exposé sur les moyens et les méthodes de la reconnaissance géotechnique, avec la note de MM. 'Gonin et Pfefer.

- La présentation d'un outil privilégié de cette reconnaissance, avec la communication de MM. Aubert et Gros, sur les photographies aériennes.

- De remarquables exemples d'aménagement urbain, dans le rapport de MM. Mahé et Fontalirand.

- Des exemples, enfin, d'aménagements littoraux dans les contributions de M. Dupain, en zone d'estuaire, et de M. Grovel, en bord de mer.

Pour tout dire, cette préoccupation d'amélioration du paysage urbain et rural n'est pas limitée à ces cinq communications : les contributions du sous-thème 1-2 sur les stockages et installations souterrains, et le sous-thème 1-3 sur les exploitations à ciel ouvert entrent tout à fait dans cette même perspective.

L'implantation souterraine est envisagée ici comme solution alternative de nature à protéger au mieux l'environnement.

Dans leur allocution d'accueil et la "prise en compte de la géotechnique et de l'environnement pour l'aménagement de l'agglomération nantaise ”, MM. A. Mahé et P. Fontalirand nous ont opportunément rappelé le cas de la ville de Nantes et de son implantation, il y a plus de 3000 ans, à la confluence de la Loire et de l'Erdre, à l'initiative de précurseurs de l'analyse sitologique. Nul doute que ses fondateurs ont procédé, avant la lettre, à une analyse géomorphologique du site, mettant en évidence le franchissement aisé du fleuve grâce à la présence de nombreuses illes.

Après un grand bond dans I'Histoire, un autre fait marquant, suite aux destructions causées par la Seconde Guerre mondiale, concerne la priorité donnée dans un premier temps au $\propto$ quantitatif $»$, pour reloger les habitants. Depuis près d'une décennie, c'est l'aspect *qualitatif * qui est mis en avant par les élus nantais, avec une politique volontariste de meilleure intégration des ouvrages dans le paysage urbain.

Dans les exemples cités, la démarche est tout à fait symptomatique : à la réflexion, on réalise, tout comme $M$. Jourdain faisait de la prose, que la géotechnique était directement concernée dans tous ces travaux d'aménagement.

Parmi les cas évoqués ici (remblaiement de zones marécageuses affectées à la construction et aux zones de loisirs, aménagement d'anciennes carrières, ouvrages d'art comme le pont des Bourdonnières ou celui de Cheviré), l'un d'eux me paraît particulièrement révélateur de cette tendance, avec la prise en compte croissante de l'environnement et de critères esthétiques pour l'orientation des projets : dans les Z.U.P. de Beaulieu et de Pirmil, le site et les caractéristiques géomécaniques médiocres des sols imposent pratiquement l'alternative de fondations prfondes, pour des surcharges importantes, ou de fondations superficielles grâce à un allègement des superstructures. C'est cette dernière orientation qui prévaut actuellement dans l'achèvement de la Z. U.P. de Beaulieu, avec un plan d'épannelage permettant de construire des immeubles bas beaucoup mieux intégrés dans le paysage et beaucoup plus conformes aux aspirations de la population.

Je pense enfin que nous partageons tous la conclusion de MM. Mahé et Fontalirand pour ce qui concerne la nécessaire intervention des spécialistes des sols et des roches, très en amont des projets, pour une meilleure évaluation des contraintes naturelles.

MM. H. Gonin et D. Pfefer, dans la communication intitulée «Problèmes de sol et d'environnement en région nantaise $n$ passent en revue les méthodes et les moyens de la reconnaissance des sols.

La démarche est exemplaire, puisqu'elle se propose d'abord de décrire l'état du site à aménager, puis d'étudier sa genèse et de se préoccuper des modifications que l'on envisage d'y apporter.

Parmi ces moyens et ces outils, la place de la géomorphologie, de la géologie, de la photointerprétation, des procédés géophysiques, de l'analyse structurale, de l'hydrogéologie et de la mécanique des sols sont rappelées à partir d'exemples généraux.

Le cas de la traversée du marais de Gesvres par la rocade Nord de la ville de Nantes fait bien prendre conscience des difficultés du site: le schiste, qui constitue localement le bed-rock, est surmonté par une couche de vase organique et de tourbe susceptible de dépasser $15 \mathrm{~m}$ d'épaisseur. On est amené à partager le regret des auteurs, qui n'ont pu réaliser la campagne sismique projetée, lorsqu'on considère le fort contraste de vélocité des ondes sismiques entre les sédiments de couverture et le rocher sous-jacent et la détermination à priori aisée de la conformation topographique du substratum rocheux à partir des dromochroniques.

La conclusion de ce travail, qui évoque ce que devrait être le contenu du rapport d'étude de sols, me paraît à même d'alimenter notre discussion, tout à l'heure, à l'occasion du débat général sur le thème I.

Un outil privilégié de cette étude de sols fait l'objet de la communication de MM. J. Aubert et G. Gros sur « la photographie aérienne pour la reconnaissance de l'environnement" .

Intervention tout à fait en amont des projets et analyse systématique de couvertures photo-aériennes exécutées à différentes époques, sur un même site, sont à préconiser pour appréhender globalement ce site et pour l'orientation des campagnes de reconnaissance géotechnique.

Une zone d'expérimentation d'environ $40 \mathrm{~km}^{2}$, dans le département de l'Yonne, est ainsi étudiée à partir de couvertures-photo exécutées entre 1946 et 1976.

Des prises de vues obliques à basse altitude, en utilisation conjointe avec les couples stéréo, permettraient un allègement notable de la campagne de reconnaissance géotechnique.

Nous aurons certainement, au moment des discussions, le point de vue des bureaux d'études sur cette 
simplification et peut-être sur le coût ainsi réduit de l'étude géotechnique.

En conclusion, l'étude de l'environnement facilite le choix des outils et le dimensionnement de l'étude géotechnique. L'utilisation des photos aériennes est caractéristique du sens de la démarche du géologue. au-delà de l'emprise stricto sensu de l'ouvrage ou du projet.

II est regrettable par ailleurs de noter encore trop souvent, de la part du Maître d'Ouvrage, une réticence pour passer commande d'une couverture spéciale à grande échelle, à l'occasion des projets importants. Si le temps très minuté le permet, quelques clichés vous seront présentés en fin de séance, à partir de couvertures à une échelle voisine du $1 / 1000$, en noir et blanc et sur émulsion infra-rouge couleur, en vue de la résolution de problèmes spécifiques de fontis, dans une zone industrielle près d'Argenton-sur-Creuse.

Dans sa note sur les « effets de l'endiguement de la rive nord de l'estuaire de la Loiren, M. R. Dupain décrit l'alluvionnement consécutif aux travaux exécutés entre 1930 et 1979 sous la direction du Port Autonome de Nantes-Saint-Nazaire.

La stabilisation du chenal de navigation s'est accompagnée, dans la concavité de Montoir, du dépôt de vase très plastique et compressible, d'environ $5 \mathrm{~m}$ d'épaisseur.

Deux cas de rupture de sol après chargement sont étudiés :

- sur la route d'accès au terminal roulier,

- sur l'aire de stockage des phosphates de Stocaloire.

On notera que les essais œdométriques ne montrent pratiquement aucune amélioration après quatre ans de stockage (ce que d'aucuns pourraient interpréter comme un fluage latéral sous charge). Cette évolution générale du site était prévisible, mais la réalisation de zones industrielles n'était pas le but recherché en 1930 , à l'origine du projet.

La dernière communication du sous-thème 1 vient de M. A. Grovel. Elle concerne «l'environnement et la pose d'une conduite sous-marine" de $2300 \mathrm{~m}$ de longueur et de $250 \mathrm{~mm}$ de diamètre, près de Lorient, dans une région particulièrement exposée à la houle.

L'étude préliminaire, réalisée par l'auteur de la communication, avait pour finalité la définition des contraintes d'environnement, au niveau des choix du tracé, de la conception et de la mise en œuvre.

La solution adoptée de conduite ensouillée, orientée perpendiculairement à la houle dominante, s'est révélée bien adaptée aux difficultés du site.

L'étude de l'environnement et les mesures pratiquées, après exécution de l'ouvrage, débouchent sur une

conclusion très pratique: une bonne protection doit être assurée, dans un environnement comparable, à partir d'enrochements déversés de part et d'autre d'une conduite sous-marine.

Dans le cadre du thème 1 entre également une communication orale de M. C. Burlet (SERALP, Lyon), intitulée: "Influence de l'environnement sur la conception et l'exécution des ouvrages et des travaux de la manufacture d'Annecy."

Cette opération de Rénovation Urbaine a été engagée par la municipalité d'Annecy sous la forme d'un concours d'architecture.
L'emplacement du projet occupait un site particulièrement privilégié, au carrefour de la vieille ville et de la ville moderne.

Pour l'insertion, dans un tel site, de plus de $10000 \mathrm{~m}^{2}$ de constructions neuves, le lauréat du concours prit le parti de réaliser une architecture très fondue dans le contexte et proiongeant le bâti existant.

Deux conséquences principales en découlèrent au niveau technique :

- la réalisation d'un minimum de deux niveaux en sous-sol, pour réduire le volume des superstructures et assurer le stationnement des véhicules automobiles,

- la recherche de structures permettant de rationnaliser, dans les sous-sols et fondations, les formes et les volumes complexes des bâtiments.

La qualité très médiocre du sous-sol a considérablement amplifié ces contraintes d'environnement et a exigé des études approfondies pour mener, dans les meilleures conditions de sécurité et de prix, l'ensemble des travaux d'infrastructure.

Les principales difficultés rencontrées résultaient:

- des caractéristiques géomécaniques très médiocres des terrains d'assise,

- des contraintes hydrauliques induites par la proximité de la rivière Thiou,

- de la vétusté des immeubles riverains, dont le caractère historique ne permettait pas la destruction. et dont les fondations étaient très mal connues,

- de l'obligation de respecter un prospect très strict. avec conservation des vues existantes sur le château et les toitures environnantes, imposant les profondeurs de terrassement.

La coupe géologique, sur le site, montre une épaisseur moyenne de remblai de l'ordre de $3 \mathrm{~m}$, recouvrant une argile silteuse grise de consistance plastique avec une teneur en eau de 30 à $40 \%$, voisine de la limite de liquidité. L'angle de frottement interne se situe, suivant les essais, de $4^{\circ}$ à $8^{\circ}$, et la cohésion varie de 0,6 à 1,1 bar.

Les essais pénétrométriques font ressortir une résistance en pointe quasiment nulle avec, dans l'emprise de l'opération, le toit du bed-rock situé entre $-5 \mathrm{~m}$ et $-55 \mathrm{~m}$ de profondeur.

Au vu des caractéristiques du sol et de sa géométrie, l'objectif des concepteurs a été de réaliser une " boîte $"$, dans un terrain se comportant pratiquement comme un liquide visqueux.

Pour assurer la stabilité, tant en phase provisoire qu'en phase définitive, on a envisagé une enceinte étanche à base de parois moulées et de palplanches, avec, localement, butonnage et ancrage par tirants.

La mise en place des palplanches, prévue à l'origine par vibration, a été, en définitive, effectuée par battage: le premier procédé provoquait en effet des nuisances beaucoup plus importantes du fait de phénomènes vibratoires se répercutant dans les immeubles environnants.

Les risques de sous-pression hydrostatiques ont également été à l'origine de modifications de la conception initiale des ouvrages, le niveau de la nappe phréatique se situant à $-1,50 \mathrm{~m}$ en moyenne par rapport au niveau du terrain initial.

En dépit des difficultés d'exécution, la solution par pieux fondés au rocher a finalement été retenue. 
Les précautions prises, tant au niveau des études que pendant l'exécution, ont permis de mener le chantier sans incident majeur et avec des nuisances réduites pour les riverains, et on peut considérer que l'inscription de l'opération dans le site s'est faite sans trop dénaturer le caractère historique de l'environnement bâti, en grande partie grâce aux volumes enterrés et aux techniques mises en œuvre, malgré un contexte géologique très contraignant et à priori difficile à maîtriser.

\section{Conclusion}

L'éveil ou l'émergence environnementaliste de la dernière décennie, renforcée par la crise de l'énergie, vient d'abord de la croissance des hommes, en nombre et en mobilité, sur une superficie qui reste inchangée, avec des ressources naturelles qui vont en s'amenuisant.

La recherche qui s'ensuit, pour une amélioration du cadre de vie, met en évidence tout un réseau de relations complexes et d'interactions entre l'homme et ce qui l'entoure.

En fait, le concept d'«Environnement » est presque devenu aujourd'hui un concept philosophique, plutôt qu'une entité visible et démontrable.

Dans cette recherche pour une meilleure qualité de l'environnement urbain et rural, géologues, mécaniciens des sols et mécaniciens des roches ont leur contribution à fournir. La géologie de l'aménagement est d'abord et nécessairement une géologie de l'environnement.

Étendre sa finalité jusqu'à prendre en compte l'aspect esthétique des paysages est sans doute une démarche plus risquée. Mais n'est-ce pas Karl Terzaghi qui écrivait déjà, il y a plus d'un demi-siècle : "La force directrice est l'enthousiasme, la volonté passionnée de réussir, et le résultat est la beauté, beauté de la pensée ou de la forme, parce que la beauté dans le sens le plus large du mot est la manifestation physique parfaite d'une réalité non physique..." ${ }^{(1)}$.

En définitive, l'entreprise amorcée par les spécialistes des sols et des roches n'est pas toujours aisée, ni ses processus d'intervention évidents.

II reste que cette contribution à l'amélioration de I'environnement existe et qu'elle se développe tous les jours, comme l'attestent les communications et les travaux qui nous sont présentés, avec une mention particulière pour la qualité et le nombre des entreprises et des bureaux d'études venus exposer leur matériel ou leur savoir-faire à ces journées nationales de Nantes.

(1) Extrait d'un manuscrit retrouvé dans les papiers de K. Terzaghi, à sa mort. Cette note, sur le thème de $\propto$ La vie et l'existence *, est datée du 31 décembre 1923 (Istanbul). 